\title{
Generic Digitalisation
}

\section{Dear Reader,}

Back in 1990, when the most advanced feature of a new medium speed engine was its electronic monitoring and diagnostics system, an obvious question at the official press conference, was "with all these sensors installed, why don't you fit electronic fuel injection?" The answer lay in the challenges posed by heavy fuel, because digital governing was already in use on some high speed engines - in fact, it was on gas engines that electronics first made their large engine mark.

A quarter of a century later, what started with fuel injection now encompasses not just the control of most engine systems but complete engine-powered systems, both marine and land-based and both stationary and mobile. And the potentials for optimisation and increases in system productivity are correspondingly immense.

As emphasised by the papers and presentations at the CIMAC Congress in Helsinki, digitalisation will be critical in the process of system integration as a means of extracting further major gains in efficiency and environmental compatibility from ships and power stations.

At a time when great advances have been consolidated and margins of improvement on the engine side can be expected to be in low single digit percentages, equal or greater onus is now rightly on mechanical, electrical, hydraulic and pneumatic propulsion and working systems to maximise energy efficiency. Likewise specialists in control and automation are called upon to perfect the interaction of all the systems in a ship or cogeneration plant, for example.

Paradoxically, it is precisely the sensors whose use in monitoring technology journalists found unambitious in 1990 that can deliver immense progress in optimisation. The point was made several times in Helsinki that other sectors of industry are further down the road in harnessing Big Data. But the large engine industry does not have to look far. Just before the Congress ABB opened its second facility to remotely monitor its Azipod steerable propulsion units on passenger, cargo and ice-going vessels (can ABB turbochargers be far behind?). And since the CIMAC Congress we have become aware of OEMs of mobile equipment - and even their component suppliers - who already offer open, generic, off-the-shelf solutions for remote data acquisition. Systems which as standard include a range of connectivity, online data processing and features employing global positioning and tracking.

The interesting terms are "open” and "generic". Users of engines with CANBus electronics do not have to wait for engine builders to implement remote data management on their behalf. On the other hand, only engine builders and systems suppliers will be able to offer the special functions and longerterm gains in productivity that come from round-the-clock engine and power systems surveillance.

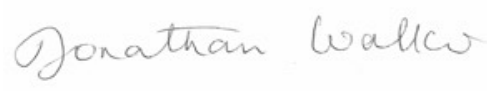

\section{Jonathan Walker}

Chief Correspondent, MTZindustrial

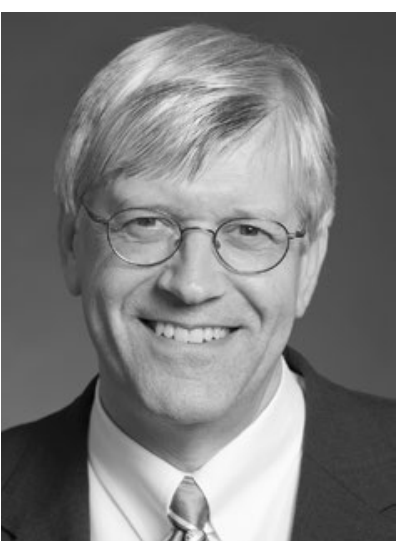

ATZ we

\section{Spotlight \\ on Powertrain and \\ Vehicle Engineering.}

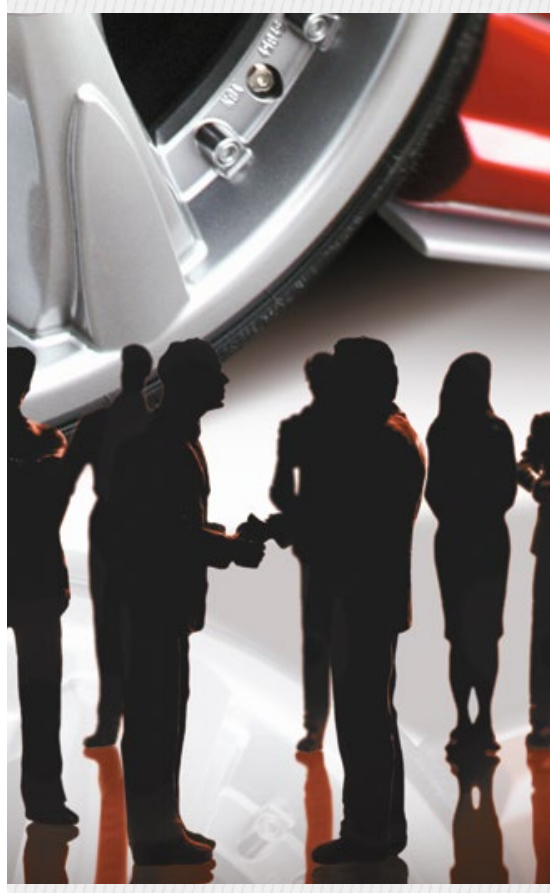

CONFERENCES FOR VEHICLE AND ENGINE SPECIALISTS

- Whole Vehicle

- Engine and Powertrain

- Chassis and Driver Assistance

- Bodywork and Automotive Acoustics

- Electric Mobility

CURRENT CONFERENCE PROGRAMS

www.ATZlive.com 